\title{
DOSAGEM DE LÍPIDES EM LIPOPROTEÍNAS PLASMÁTICAS \\ DE FRANGOS DE CORTE ALIMENTADOS COM SORGO E/OU ÁCIDO TÂNICO
}

\author{
DIAS, Luciana Thie Seki ${ }^{1}$ \\ MACHADO, Célio Raimundo ${ }^{2}$ \\ SEKI, Meire Christina ${ }^{3}$ \\ SILVA, Janaina Della Torre da ${ }^{4}$
}

Recebido em: 2009-05-21

Aprovado em: 2009-08-17

ISSUE DOI: $10.3738 / 1982.2278 .244$

RESUMO: O objetivo do trabalho foi avaliar a influência de dietas com sorgo em substituição ao milho e adição de ácido tânico nas rações à base de milho e soja sobre o colesterol e triglicérides das lipoproteínas plasmáticas e do plasma total, em frangos de corte. Após obtenção do plasma das aves, as lipoproteínas de muito baixa densidade (VLDL), de baixa densidade (LDL) e de alta densidade (HDL), foram separadas em ultracentrífuga por gradientes de densidade. Após a ultracentrifugação por 20 horas a $8^{\circ} \mathrm{C}$ a $25.000 \mathrm{rpm}$, coletaram-se as lipoproteínas e de cada uma destas, dosou-se os níveis de colesterol e triglicérides. Não se observaram diferenças estatísticas significativas entre os tratamentos, portanto dietas com sorgo em substituição ao milho e com adição de ácido tânico nas rações à base de milho e soja não alteram significativamente os níveis de colesterol e triglicérides nas lipoproteínas e no plasma total.

Palavras-chaves: Frangos de corte. Ultracentrifugação. Sorgo. Ácido tânico. Lipoproteínas

\section{DOSAGE OF LIPIDS IN PLASMA LIPOPROTEINS OF BROILERS FEEDS WITH SORGHUM AND/OR TANIC ACID}

SUMMARY: This study evaluated the effects of sorghum in corn substitution and tanic acid added to corn-soybean based diets on plasma cholesterol levels and triglyceride levels in lipoprotein fractions and total plasma in broilers. Lipoproteins of very low density lipoprotein (VLDL), low density (LDL) and high density

(HDL) of the plasma were separated by ultracentrifugation using density gradient at $25.000 \mathrm{rpm}$ for 20 hours and $8^{\circ} \mathrm{C}$. Lipoproteins were collected and cholesterol and triglycerides were determined in each fraction. Not observed significant differences between treatments. Therefore, the substitution of corn by sorghum and the addition of tanic acid to corn-soybean based diets given to broilers did not change significantly cholesterol and triglyceride levels in lipoprotein fractions and in total plasma.

Keywords: Broilers. Ultracentrifugation. Sorghum. Tanic acid. Lipoprotein

\footnotetext{
${ }^{1}$ Professora Adjunto do CCA/UFSCar, Rodovia Anhanguera, km174 CP 153 Araras - SP - Brasil, CEP13600-970, e-mail: sekidias@cca.ufscar.br

${ }^{2}$ Professor Titular da FCAV/UNESP, Via de Acesso Prof. Paulo Donato Castellane, s/n, Jaboticabal - SP - Brasil, CEP 14884-900, e-mail: cmachado@fcav.unesp.br

${ }^{3}$ Doutoranda do Programa de Medicina Veterinária com Concentração em Patologia Animal da FCAV/UNESP, Via de Acesso Prof. Paulo Donato Castellane, s/n, Jaboticabal - SP - Brasil, CEP 14884900, e-mail: meireseki@ hotmail.com

${ }^{4}$ Doutoranda do Programa de Zootecnia da FCAV/UNESP, Via de Acesso Prof. Paulo Donato Castellane, s/n, Jaboticabal - SP - Brasil, CEP 14884-900, e-mail: janainadts@ hotmail.com
} 


\section{INTRODUÇÃO}

$\mathrm{Na}$ indústria avícola brasileira, o segmento de frangos de corte foi o que mais se desenvolveu nas duas últimas décadas. Além de abastecer o mercado interno com proteína animal de alta qualidade e gerar direta e indiretamente considerável número de empregos, consegue resultados econômicos altamente satisfatórios com a exportação de produtos avícolas para diversos países em todo o mundo.

Junto a este crescimento de produção, desenvolveram-se técnicas de maximização do crescimento do frango de corte e a indústria avícola tem obtido sucesso produzindo frangos maiores em menor tempo (HAVESTEIN et al, 1994). Mas este frango maior apresenta o inconveniente de depositar maior quantidade de gordura na carcaça e a indústria avícola tornou-se interessada em oferecer um produto com menos gordura para atender à demanda do público consumidor. Com isto, além de prover ao consumidor um produto mais saudável, a redução de gordura também pode reduzir os custos de processamento. Estes custos de processamento são em relação à gordura abdominal que é retirada, em sua maior parte, durante a evisceração. A gordura residual que ainda permanece é removida posteriormente pelos processadores para que as carcaças apresentem melhor qualidade e aparência. Até mesmo a gordura subcutânea, mais difícil de ser removida das carcaças, não apresenta utilidade visto ser retirada ou se desfazer pelo calor ao ser preparada. Além disso, com a crescente tendência de melhor aproveitar as carcaças, usando-as como fontes de carne para outros produtos, mesmo a gordura subcutânea é perdida totalmente durante o processo de retirada do músculo. A redução da gordura nos frangos de corte auxiliaria a diminuir os custos de limpeza e problemas de poluição associados à água de limpeza dos abatedouros. Sem contar que a deposição de gordura é energeticamente ineficiente, pois o tecido magro requer menos energia por unidade de peso vivo do que a gordura, devido à menor densidade energética da proteína (CARSTENS, 1995).

O processo lipogenético em aves apresenta características específicas. No frango jovem, cerca de $80-85 \%$ dos ácidos graxos que se acumulam no tecido adiposo são derivados dos lipídios produzidos no fígado. A razão para isso é devido as atividade das enzimas lipogenéticas no tecido adiposo serem muito baixas, deslocando-se ou sendo no fígado o principal sítio de lipogênese (O’HEA; LEVEILLE, 1969, 1975; SAADOUN; LECLERCQ, 1983). A maior parte dos ácidos graxos contidos nas lipoproteínas da ave é derivada da dieta ou sintetizada de carboidratos no fígado e a sua posterior absorção é 
fator determinante no crescimento do tecido adiposo. Dos vários tipos de lipídios produzidos no fígado de aves, o mais importante é a lipoproteína de muito baixa densidade (VLDL) porque se constitui na principal fonte de ácidos graxos a partir da qual os adipócitos podem produzir e depositar os seus próprios lipídios. A relação positiva entre a VLDL transportada pelo plasma e a adiposidade corporal em frangos sugere que tal situação depende da disponibilidade do substrato lipídico para a captação pelo adipócito. Assim sendo, as elevadas concentrações plasmáticas de lipoproteínas em aves obesas não resultam de um ritmo lento de captação pelo adipócito, mas de um aumento na sua síntese e secreção pelo fígado (LEGRAND et al, 1987; SAADOUN; LECLERCQ, 1987; GRIFFIN et al, 1991).

A concentração de VLDL na circulação parece ser o principal fator causador da adiposidade porque apresenta uma correlação positiva com o conteúdo das gorduras abdominal e total (GRIFFIN, 1981; WHITEHEAD; GRIFFIN, 1982; GRUNDER; CHAMBERS, 1985). Prova disso foram as seleções genéticas para baixas concentrações de VLDL plasmática, resultando em aves com quantidades consideravelmente menores de gordura (WHITEHEAD; GRIFFIN, 1984).

A fração da lipoproteina que está diretamente relacionada com a quantidade de gordura armazenada no organismo é os triacilgliceróis. O TAG é o lipídio predominante nos portomícrons e na VLDL ( 88 e 56\% da molécula, respectivamente), enquanto que o colesterol e fosfolipídios são predominantes nas lipoproteinas de baixa (LDL) e alta densidade (HDL), (48 e 46\%, respectivamente). Assim sendo, as determinações dos TAG dos portomícrons e VLDL refletem o fluxo de lipídios originários do intestino e do fígado.

De acordo com Behr et al (1981), a LDL é derivada de VLDL de origem hepática, após a mesma ter sofrido a ação da lipase lipoprotéica. Além disso, alguns dos constituintes de superfície da VLDL são também transferidos para a HDL. Assim se a atividade da lipase lipoprotéica for inibida, aproximadamente $80 \%$ das LDL circulantes e $50 \%$ das HDL desaparecerão dentro de 8 horas.

A alimentação é responsável por $65 \%$ do custo de produção de frangos de corte, assim, pesquisas vêm sendo conduzidas no sentido de se maximizar a utilização de insumos disponíveis no mercado interno, objetivando sempre a diminuição nos custos de produção, desta forma a cultura de sorgo tem a vantagem de ser mais tolerante às adversidades do tempo, sem comprometer os conteúdos nutritivos que são bem semelhantes ao do milho, cereal largamente utilizado na formulação de rações para 
aves, onde participa como o principal ingrediente energético. Comparativamente, o sorgo apresenta de 90 a 95\% do valor nutritivo do milho (WHITAKER; CARVALHO, 1997).

Existem duas classes de taninos, os hidrolizáveis e os condensados. Os taninos hidrolizáveis, como o ácido tânico, decompõem-se em açúcares e ácidos fenólicos (ácido gálico ou elágico) quando tratados com ácidos, bases, ou alguma enzima hidrolítica. No sorgo encontra-se predominantemente o tanino condensado, um polímero resultante da condensação de unidades de flavan-3 ol, denominado de proantocianidina.

Os taninos são capazes de reagir com proteínas, polissacarídeos e outras moléculas, e ainda atuarem como quelantes de minerais. Podem também inibir a ação de enzimas digestivas ou terem a produção de mucina alteradas na presença de taninos e reduzir a digestibilidade de aminoácidos e da energia da dieta. É por estas razões que a presença de tanino nos alimentos tem alguns efeitos detrimentais na saúde e no desenvolvimento animal, incluindo a depressão na palatabilidade do alimento e ingestão voluntária, digestibilidade da proteína, carboidratos, do amido, lipídios e diminuição na absorção de cálcio.

Recentemente os estudos sobre nutrição de aves apresentam considerável interesse no acompanhamento dos níveis de lipídios, triacilgliceróis e colesterol, além dos ácidos graxos, como forma de estimar algumas condições fisiológicas das aves.

Um efeito do tanino sobre a deposição de gordura em aves foi verificado por Pour-eza; Edriss (1997). Os autores encontraram uma correlação positiva e altamente significativa entre a ingestão de taninos e a porcentagem de gordura abdominal em frangos de corte, e sugeriram que esta correlação poderia resultar dos efeitos dos taninos sobre a digestibilidade das proteínas, e sua conseqüente redução na disponibilidade de aminoácidos. Assim, com a diminuição na síntese protéica, uma maior parte da energia fornecida na direta seria depositada na forma de gordura. Mas Bartov (1985) utilizandose de injeções de corticosterona em frangos com dietas de milho ou sorgo, não foram observadas diferenças no acúmulo de gordura na carcaça ou mesmo no fígado aos 28 dias de idade.

Sabe-se que hoje o setor avícola enfrenta sérios problemas gerados pela elevada velocidade de crescimento apresentada pelas aves. Estes animais tornam-se muito susceptíveis à ocorrência de doenças metabólicas e desarranjos esqueléticos que levam a problemas de perna. Além disso, o elevado acúmulo de gordura verificado nas carcaças 
é um aspecto a ser considerado, tanto do ponto de vista econômico quanto da saúde do consumidor. Os diversos tipos de alimentos e suas proporções nas rações influenciam as concentrações de lipoproteínas, mas os estudos nas aves a esse respeito são ainda insuficientes. De maneira semelhante é pouco conhecido o papel do jejum no fornecimento de precursores para a lipogênese hepática.

Em vista do exposto, o estudo dos mecanismos da síntese das lipoproteínas e as características das atividades da lipase lipoprotéica constituem-se pontos importantes na abordagem das causas da adiposidade aumentada assim como a influência de dietas com sorgo em substituição ao milho e com adição de ácido tânico nas rações a base de milho e soja em aves em estado alimentado sobre as concentrações plasmáticas de triacilgliceróis e colesterol nas lipoproteínas, objetivo deste trabalho.

\section{MATERIAL E MÉTODOS}

\section{Animais e seu tratamento}

O experimento foi conduzido em biotério climatizado com temperatura de $22 \pm$ $3^{\circ} \mathrm{C}$, no Departamento de Tecnologia da FCAV/UNESP - Jaboticabal (SP), com duração de 43 dias. A linhagem utilizada foi a Cobb, com pintos machos de um dia. Foram alojadas 3 aves por gaiola, e cada gaiola foi equipado com um bebedouro e um comedouro tipo calha, fixado às gaiolas, externamente. Os bebedouros foram lavados diariamente e os comedouros tinham ração à vontade.

As rações, isoprotéicas e isoenergéticas, foram fornecidas ad libitum durante todo o período experimental. Foram fornecidos dois tipos de ração, uma inicial (1-21 dias) e uma de crescimento (22-43 dias). Os balanceamentos das rações utilizadas (Tabela 3) seguiram as tabelas de composição de ingredientes e exigências nutricionais propostas por Rostagno et al (1994). Tomou-se o devido cuidado para que todas as rações tivessem os mesmos ingredientes, pois a adição ou retirada de um ou mais ingredientes poderia ocasionar mudanças no perfil de ácidos graxos e conseqüentemente na resposta fisiológica dos lipídios que foram estudados. 
Tabela 1. Composição percentual e calculada das rações fornecidas às aves nos períodos iniciais (1 a 21 dias) e de crescimento (22 a 42 dias).

\begin{tabular}{|c|c|c|c|c|c|c|c|c|c|c|}
\hline \multirow{2}{*}{$\begin{array}{c}\text { Ingredientes } \\
(\%)\end{array}$} & \multicolumn{5}{|c|}{ Inicial } & \multicolumn{5}{|c|}{ Crescimento } \\
\hline & CONT & SBT & SAT & BAT & AAT & $\begin{array}{c}\mathrm{CON} \\
\mathrm{T}\end{array}$ & SBT & SAT & BAT & AAT \\
\hline Milho moído & 52,13 & 36,39 & 35,66 & 52,13 & 52,13 & 55,20 & 39,47 & 38,74 & 55,20 & 55,20 \\
\hline Farelo de soja & 40,71 & 40,75 & 40,86 & 40,71 & 40,71 & 33,56 & 33,59 & 33,70 & 33,56 & 33,56 \\
\hline Óleo vegetal & 2,16 & 2,86 & 3,48 & 2,16 & 2,16 & 6,24 & 6,94 & 7,56 & 6,24 & 6,24 \\
\hline Núcleo ${ }^{(1)}$ & 5,00 & 5,00 & 5,00 & 5,00 & 5,00 & 5,00 & 5,00 & 5,00 & 5,00 & 5,00 \\
\hline Sorgo AT & 0,00 & 0,00 & 15,00 & 0,00 & 0,00 & 0,00 & 0,00 & 15,00 & 0,00 & 0,00 \\
\hline Sorgo BT & 0,00 & 15,00 & 0,00 & 0,00 & 0,00 & 0,00 & 15,00 & 0,00 & 0,00 & 0,00 \\
\hline Total & 100 & 100 & 100 & 100 & 100 & 100 & 100 & 100 & 100 & 100 \\
\hline \multicolumn{11}{|c|}{ Valores Calculados } \\
\hline $\mathrm{PB}(\%)$ & 23,00 & 23,00 & 23,00 & 23,00 & 23,00 & 20,00 & 20,00 & 20,00 & 20,00 & 20,00 \\
\hline EM (Mcal/kg) & 2,90 & 2,90 & 2,90 & 2,90 & 2,90 & 3,20 & 3,20 & 3,20 & 3,20 & 3,20 \\
\hline P total $(\%)$ & 0,70 & 0,70 & 0,69 & 0,70 & 0,70 & 0,67 & 0,67 & 0,66 & 0,67 & 0,67 \\
\hline P disponível (\%) & 0,46 & 0,46 & 0,46 & 0,46 & 0,46 & 0,45 & 0,45 & 0,45 & 0,45 & 0,45 \\
\hline Cálcio (\%) & 1,04 & 1,04 & 1,04 & 1,04 & 1,04 & 1,01 & 1,01 & 1,01 & 1,01 & 1,01 \\
\hline Metionina (\%) & 0,45 & 0,43 & 0,43 & 0,45 & 0,45 & 0,41 & 0,39 & 0,38 & 0,41 & 0,41 \\
\hline Lisina (\%) & 1,29 & 1,28 & 1,29 & 1,29 & 1,29 & 1,09 & 1,09 & 1,09 & 1,09 & 1,09 \\
\hline Met + Cis $(\%)$ & 0,87 & 0,86 & 0,86 & 0,87 & 0,87 & 0,78 & 0,78 & 0,78 & 0,78 & 0,78 \\
\hline
\end{tabular}

CONT - aves alimentadas com ração à base de milho e farelo de soja; SBT e SAT- adição de 15\% de sorgo, com baixo e alto teor em taninos na ração, respectivamente; BAT e AAT - adição de ácido tânico em ração à base de milho e farelo de soja, obtendo os mesmos valores percentuais de tanino do SBT e do SAT.

(1) Núcleo, níveis/kg do produto: 70.000UI (Vit. A), 40.000 UI (Vit D3), 400 UI (Vit. E), 50mg (Vit K3), 560mg (Vit. B12), 40mg (Vit B1), 72mg (Vit B2), 70mg (Vit B6), 500mg (ác. pantotênico), 710 mg (ác. nicotinico), 12mg (ác. fólico), 3mg (biotina), 9,6g (cloreto colina), 28g (metionina), 2g (lincomix), 590mg (niacina), 20g (antioxidante), 1127mg (ferro), 200mg (cobre), 1248mg (manganês), 800mg (zinco), 12,4mg (iodo), 4,05mg (selênio), 4,10mg (enxofre), 174,623g (calcário), 69,3g (fósforo), 23,4g (sódio) e veículo.

\section{Parâmetros avaliados}

Aos 43 dias de idade, coletou-se o sangue de 15 aves por tratamento. Após a coleta, o sangue foi centrifugado a $3.000 \mathrm{rpm}$ por 10 minutos para a obtenção do plasma, e estes armazenados em tubos do tipo eppendorf, mantidos a $-20^{\circ} \mathrm{C}$, até o momento da análise.

As lipoproteínas, VLDL, LDL e HDL, foram separadas em ultracentrífuga por gradientes de densidade conforme metodologias propostas por Havel et al (1955) e 
Redgrave et al. (1975); no Laboratório de Imunoparasitologia do Departamento de Patologia Animal da FCAV/UNESP - Jaboticabal (SP). Soluções de $\mathrm{NaCl}$ de diferentes densidades foram elaboradas a partir de uma solução estoque que continha $153 \mathrm{~g}$ de $\mathrm{NaCl}$ e $354 \mathrm{~g}$ de $\mathrm{KBr} / \mathrm{L}$ com densidade de 1,392 e de uma solução salina de 0,15M $(13,09 \mathrm{~g} \mathrm{NaCl} / \mathrm{L})$ e densidade de 1,005 .

Num tubo próprio para ultracentrífuga modelo Beckman Optima LE-80K com rotor SW 28.1, colocou-se 3,5 mL de plasma. Esta quantia se refere a um "pool" de 3 amostras de plasma de um mesmo tratamento e coletadas no mesmo horário. Juntamente com o plasma adicionou-se $1,14 \mathrm{~g}$ de $\mathrm{KBr}$ e $0,5 \mathrm{~mL}$ da solução com densidade de 1,210. Logo em seguida acrescentou-se lentamente 2,5mL da solução com densidade de 1,065; 2,5 mL da solução com densidade de1,020 e $2 \mathrm{~mL}$ da solução com densidade de 1,005 (solução salina) .

As amostras foram ultracentrifugadas durante 20 horas a $8^{\mathrm{O}} \mathrm{C}$ a $25000 \mathrm{rpm}$. Após a ultracentrifugação coletou-se as lipoproteínas, e acondicionou-se em tubos tipo eppendorf para posterior análises de triacilgliceróis e colesterol total.

Os triacilgliceróis das lipoproteinas foram quantificados pelo método proposto por Fossati (1982), McGowan et al (1983) e Nagele et al (1984) por meio de "kit" (Cat. 59, LABTEST Diagnóstica S.A., Belo Horizonte, MG) no Laboratório de Patologia Clínica do Hospital Veterinário da FCAV-UNESP - Jaboticabal (SP).

O colesterol total plasmático foi quantificado pelo método proposto por Alain et al. (1974), modificado por Good et al (1966) por meio de "kit" (Cat. 60, LABTEST Diagnóstica S.A., Belo Horizonte, MG), no Laboratório de Patologia Clínica do Hospital Veterinário da FCAV-UNESP - Jaboticabal (SP). Os dados foram analisados como delineamento inteiramente casualizados (DIC), utilizando-se do programa SAS.

\section{RESULTADOS}

\section{Triacilgliceróis}

Os valores médios obtidos para o nível de triacilgliceróis no plasma total, VLDL, LDL e HDL não apresentam diferenças significativas entre os tratamentos. Os valores médios obtidos para o nível de triacilgliceróis estão expressos nas Tabela 1 e Figura 1. 
Tabela 2. Médias dos níveis de triacilglicerol (mg/dL) de frangos de corte aos 43 dias de idade, alimentados com rações contendo sorgo e/ou ácido tânico.

\begin{tabular}{|l|c|c|c|c|c|}
\hline TRATAMENTO & $\mathbf{n}$ & PLASMA TOTAL & VLDL & LDL & HDL \\
\hline CONTROLE & 5 & 97,74 & 83,28 & 9,80 & 5,26 \\
\hline SBT & 5 & 103,78 & 89,82 & 10,48 & 6,32 \\
\hline SAT & 5 & 108,40 & 86,22 & 11,14 & 5,70 \\
\hline AAT & 5 & 110,20 & 93,44 & 11,80 & 5,54 \\
\hline BAT & 5 & 103,60 & 89,38 & 10,76 & 5,22 \\
\hline
\end{tabular}

Controle - dieta comercial à base de milho e soja;

SBT- substituição de $15 \%$ do milho da dieta por grãos de sorgo com baixo teor em taninos;

SAT- substituição de $15 \%$ do milho da dieta por grãos de sorgo com alto teor em taninos;

AAT- adição de ácido tânico em ração comercial à base de milho e soja, até que atinja os mesmos valores percentuais de tanino do tratamento SAT (alto teor em taninos).

BAT- adição de ácido tânico em ração comercial à base de milho e soja, até que atinja os mesmos valores percentuais de tanino do tratamento SBT (baixo teor em taninos);

VLDL - lipoproteína de muito baixa densidade

LDL - lipoproteína de baixa densidade

HDL - lipoproteína de alta densidade

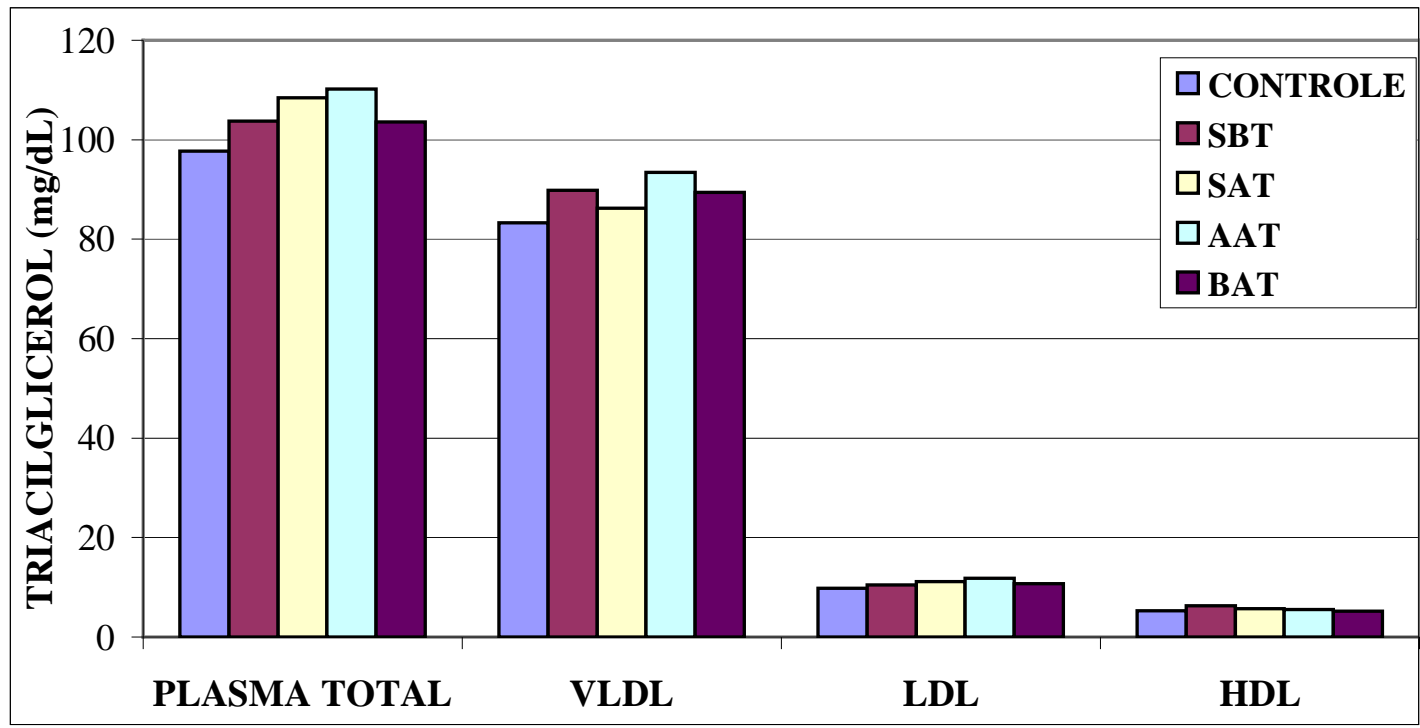

Figura 1. Médias dos níveis de triacilglicerol $(\mathrm{mg} / \mathrm{dL})$ de frangos de corte aos 43 dias de idade, alimentados com rações contendo sorgo e/ou ácido tânico.

Controle - dieta comercial à base de milho e soja;

SBT- substituição de 15\% do milho da dieta por grãos de sorgo com baixo teor em taninos;

SAT - substituição de 15\% do milho da dieta por grãos de sorgo com alto teor em taninos;

AAT-adição de ácido tânico em ração comercial à base de milho e soja, até que atinja os mesmos valores percentuais de tanino dotratamento SAT (alto teor em taninos).

BAT-adição de ácido tânico em ração comercial à base de milho e soja, até que atinja os mesmos valores percentuais de tanino do tratamento SBT (baixo teor em taninos);

VLDL - lipoproteína de muito baixa densidade

LDL - lipoproteína de baixa densidade

HDL - lipoproteína de alta densidade 


\section{Colesterol}

Com relação ao nível de colesterol no plasma total, VLDL, LDL e HDL, verifica-se que não houve diferença significativa entre os tratamentos. Os valores médios obtidos para o nível de colesterol estão expressos nas Tabela 2 e Figura 2.

Tabela 3. Médias dos níveis de colesterol (mg/dL) de frangos de corte aos 43 dias de idade, alimentados com rações contendo sorgo e/ou ácido tânico.

\begin{tabular}{|l|c|c|c|c|c|}
\hline TRATAMENTO & $\mathbf{n}$ & PLASMA TOTAL & VLDL & LDL & HDL \\
\hline CONTROLE & 5 & 102,40 & 4,68 & 67,00 & 17,66 \\
\hline SBT & 5 & 102,80 & 4,66 & 66,40 & 21,20 \\
\hline SAT & 5 & 116,72 & 4,68 & 79,46 & 24,08 \\
\hline AAT & 5 & 120,40 & 5,80 & 79,00 & 24,10 \\
\hline BAT & 5 & 105,48 & 4,42 & 65,86 & 18,62 \\
\hline
\end{tabular}

Controle - dieta comercial à base de milho e soja;

SBT- substituição de $15 \%$ do milho da dieta por grãos de sorgo com baixo teor em taninos;

SAT- substituição de $15 \%$ do milho da dieta por grãos de sorgo com alto teor em taninos;

AAT- adição de ácido tânico em ração comercial à base de milho e soja, até que atinja os mesmos valores percentuais de tanino do tratamento SAT (alto teor em taninos).

BAT- adição de ácido tânico em ração comercial à base de milho e soja, até que atinja os mesmos valores percentuais de tanino do tratamento SBT (baixo teor em taninos);

VLDL - lipoproteína de muito baixa densidade

LDL - lipoproteína de baixa densidade

HDL - lipoproteína de alta densidade

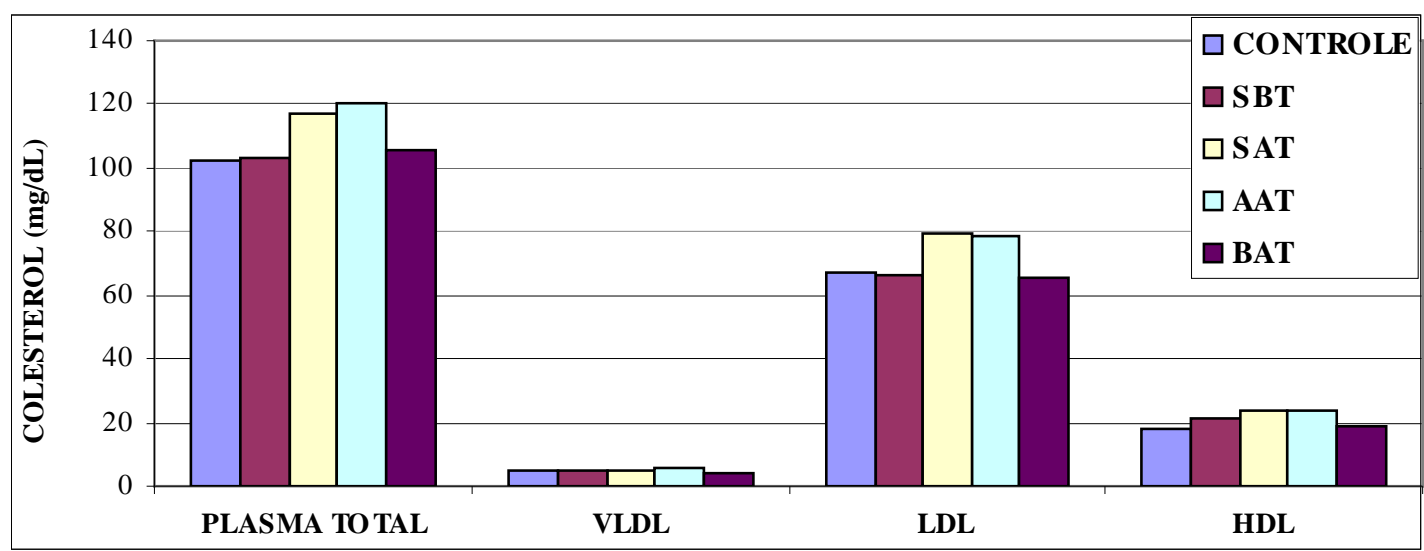

Figura 2. Médias dos níveis de colesterol (mg/dL) de frangos de corte aos 43 dias de idade, alimentados com rações contendo sorgo e/ou ácido tânico.

Controle - dieta comercial à base de milho e soja;

SBT-substituição de 15\% do milho da dieta por grãos de sorgo com baixo teor em taninos;

SAT-substituição de 15\% do milho da dieta por grãos de sorgo com alto teor em taninos;

AAT-adição de ácido tânico em ração comercial à base de milho e soja, até que atinja os mesmos valores percentuais de tanino dotratamento SAT (alto teor em taninos).

BAT-adição de ácido tânico em ração comercial à base de milho e soja, até que atinja os mesmos valores percentuais de tanino do tratamento SBT (baixo teor em taninos);

VLDL - lipoproteína de muito baixa densidade

LDL - lipoproteína de baixa densidade

HDL - lipoproteína de alta densidade 


\section{DISCUSSÃO}

Os achados obtidos no presente experimento demonstraram que dietas com sorgo em substituição ao milho e com adição de ácido tânico nas rações a base de milho e soja não induziram alterações significativas no nível de triacilgliceróis e colesterol do plasma total, da VLDL, da LDL e da HDL em aves no estado nutrido. Segundo a literatura consultada, em mamíferos, a retirada de alimento, por 2 horas, resulta na cessação completa da síntese de ácidos graxos pelo fígado (LEVEILLE et al, 1975) e decréscimos nos níveis de VLDL e HDL são observados após jejum de um dia (SHAPIRA et al, 1979).

\section{REFERÊNCIAS}

ALAIN, C. A. et al. Clinical Chemistral. v. 20, p.470, 1974.

BARTOV, I. Effect of dietary factors on the responses of chicks to corticosteron injections.British Poultry Science, v.26,p.473-81,1985.

BEHR. S. R et al. A. Plasma lipoproteins change resulting from immunologically blocked lipolysis Journal of Lipid Research, v.22, p.443-450,1981.

CARSTENS, G. E. Compensatory growth in beef cattle In: SYMPOSIUM INTAKE BY FEEDLOTCATTLE,1995,Oklahoma. Proceedings...p.70-84,1995

DIAS, L.T.S. Metabolismo hepático de lipídios em frangos de corte (Gallus domesticus) com diferentes níveis de proteína e energia na dieta. 1999. Dissertação (Mestrado em Produção Animal) - Faculdade de Ciências Agrárias e Veterinárias, Universidade Estadual Paulista, Jaboticabal.

FOSSATI, P.; PRENCIPE,L.Clinical Chemistral. v.28, p.2077, 1982

GRIFFIN, H.D; WINDSOR, D.; WHITEHEAD, C.C. Changes in lipoprotein metabolism and body composition in chickens in response to divergent selection for plasma very low density lipoprotein concentration. British Poultry Science, v.32, p.195-201, 1991

GRIFFIN, H.D.; WHITEHEAD, C.C.; BROADBENT, L.A. The relationship between plasma triglyceride concentrations and body fat content in male e female broilers - a basisforselection British Poultry Science, v.21,p.107-13,1981.

GRUNDER, A.A.; CHAMBERS, J.R. Plasma very low density lipoproteins and abdominal fat in broiler chickens: herdabilities and genetic correlations. Poultry Science, v.64, supl.11 p.109,1985. 
HAVEL, R. J.; EDER, H. A.; BRAGDON, J. H. Journal Clinical Investigation, v.34, p.1345-1353, 1955.

HARVENSTEIN,G. B. et al. Carcass composition and yield of 1991 vs 1957 broilers when fed "typical" 1957 and 1991 broilerdiets. Poultry Science, v.73,p.17951804,1994 .

LEGRAND, P. et al. Hepatic lipogenesis in genetically lean and fat chickens. In vitro studies. Comparative Biochemistry and Biophysical. n.87B, p.789-92, 1987.

McGOWAN, M.W. et al. Clinical Chemistral. v.29, p.538,1983

NAGELE, V. et al. Clinical Chemistral Biochemistral. v.22, p.165, 1984.

O'HEA,E. K.; LEVEILLE, G.A. Significance of adipose tissue and liver as sites of fatty acid synthesis in the pig and the efficiency of utilization of various substrates for lipogenesis. Journal of .Nutrition., v.99, p.338-44, 1969.

POUR-EZA, J.; EDRISS, M.A. Effects of dietary sorghum of different tannin concentrations and tallow supplementation on the performance of broiler chicks. British nPoutry Science, v.38, p.512-7,1997

REDGRAVE, T.G.; ROBERTS, D.C.K.; WEST,C. E. Separation of plasma lipoproteins by density gradient ultracentrifugation Ann Clin.Biochem, v. 65, p.42-9, 1965

ROSTAGNO, H.S.; SILVA, D.J.; COSTA, P. M. Composição de alimentos e exigências nutricionais de aves e suínos. Viçosa: Universidade Federal de Viçosa, 1983. 57p.

SAADOUN, A.; LECLERCQ, B. Comparison of in vitro fatty acid synthesis and transport in the domestic chick (Gallus domesticus). Comparative Biochemistry and Physiology, n.75B, p.641-44, 1983.

SAADOUN, A.; LECLERCQ, B. In vivo lipogenesis of genetically lean and fat chickens: effects of nutritional state and dietary fat. Journal of Nutrition, v. 28, p. 1424-28,1987.

SHAPIRA, N.; NACHTOMI, E.; NIR, I. Effects of isocaloric supplements of glucose or soybean oil on lipids in tissues and plasma lipoproteins of starved and overfed chickens. Nutritional Metabolism, v.23, p.368-83, 1979.

WHITAKER, H.M. A; CARVALHO, R.L. Substituição do milho pelo sorgo em rações para eqüinos. Revista da Sociedade Brasileira de Zootecnia, v.26, p.139-46, 1997.

WHITEHEAD, C.C.; GRIFFIN, H.D. Plasma lipoprotein concentration as an indicator of fatness in broilers: effect of age and diet. British Poultry Science, v.23, p.299305,1982 .

HITEHEAD, C. C.; GRIFFIN, H.D. Development of divergent lines of lean and fat broilers using plasma very low density lipoprotein concentration as selection criterion: the first three generations. British Poultry Science, v.25, p. 573-82, 1984. 
